\title{
The use of the Cambridge Neuropsychological Test Automated Battery (CANTAB) in neuropsychological assessment: application in Brazilian research with control children and adults with neurological disorders
}

\author{
Daniela Tsubota Roque, Rosani Aparecida Antunes Teixeira, Elaine C. Zachi and Dora F. Ventura \\ Universidade de São Paulo, São Paulo, SP, Brazil
}

\begin{abstract}
Computerized neuropsychological tests can be effective and have many benefits. This paper addresses these issues using the computer-based Cambridge Neuropsychological Test Automated Battery (CANTAB) to assess Brazilian children and adults. Preliminary normative data for Brazilian children obtained in a series of CANTAB subtests are presented together with data from adults (controls) and samples of patients with multiple sclerosis and Duchenne muscular dystrophy. The results obtained in children confirmed the normative values reported for American children (Luciana \& Nelson, 2002), indicating high applicability of the CANTAB battery for children in the evaluation of visual memory, working memory, planning, and set shifting. For multiple sclerosis patients, CANTAB revealed differences between the control and multiple sclerosis groups for all subtests employed, including visual memory, working memory, spatial memory, decision making, inhibitory control, and sustained attention. In children with Duchenne muscular dystrophy, the CANTAB tests revealed specific detriments in attention and memory. These results of the application of CANTAB in different patient groups indicate that the battery is appropriate for assessing several central nervous system diseases. Keywords: neuropsychological tests, CANTAB, children, multiple sclerosis, Duchenne muscular dystrophy.
\end{abstract}

Received 15 February 2011; received in revised form 31 August 2011; accepted 12 September 2011. Available on line 10 October 2011

\section{Introduction}

The Cambridge Neuropsychological Test Automated Battery (CANTAB) is a computerized neuropsychological assessment battery originally written and developed by Barbara Sahakian, Trevor Robbins, and colleagues at Cambridge University in the 1980s (Fray, Robbins, \& Sahakian, 1996; Luciana \& Nelson, 2002; Robbins et al., 1998; Sahakian et al., 1988). The first aim of CANTAB was to assess the patterns of cognitive decline in dementia in elderly individuals. However, it has been used in a wide variety of clinical populations (Fray et al., 1996; Levaux et al., 2007; Rasmussen, Soleimani, Carroll, \& Hodlevskyy, 2009 ) with different levels of ability and ages. It has also been employed in neuropsychological research across

Daniela Tsubota Roque, Rosani Aparecida Antunes Teixeira, Elaine C. Zachi, and Dora F. Ventura, Instituto de Psicologia, Universidade de São Paulo, São Paulo, Brazil. Rosani Aparecida Antunes Teixeira, Elaine C. Zachi, and Dora F. Ventura, Núcleo de Neurociências e Comportamento, Universidade de São Paulo, São Paulo, Brazil. Correspondence regarding this article should be directed to: Daniela Tsubota Roque, Av. Prof. Mello Morais, 1721, Bloco A - Sala D-9, CEP 05508-030, Cidade Universitária, São Paulo - SP, Brasil. E-mail: daniela.tsubota@gmail.com age groups to study the development of a set of cognitive functions (De Luca et al., 2003; Luciana \& Nelson, 2002).

CANTAB incorporates a wide variety of executive and memory tasks that are adapted for use with humans in paradigms developed from the evaluation of damage to specific brain areas in experimental animals (Lowe \& Rabbitt, 1998), especially damage to temporal and frontal cortical regions. The relationships between the scores on component tests of CANTAB and evidence of impairment in specific cognitive functions and psychiatric disorders have been demonstrated in a very extensive set of data (Hadwin, Brogan, \& Stevenson, 2005; Liotti et al., 2007; Owen, Sahakian, Semple, Polkey, \& Robbins, 1994; Pantelis et al., 1997; Palade \& Benga, 2007; Sweeney, Kmiec, \& Kupfer, 2000; Steele, Minshew, Luna, \& Sweeney, 2007; Torgersen, Helland, Flaatten, \& Wester, 2010; Yun da et al., 2011).

The CANTAB tests are administered using a computer with a touch-sensitive screen. Application of the test and feedback are given in a standardized manner (Fray et al., 1996).

Computer-administered tests are becoming more common because of the advantages offered to the neuropsychologist. Computerized administration does 
not require extensive training and limits the interaction between the subject and examiner. Responses are recorded and analyzed automatically, and variables that are quite difficult or impossible to record with manual administration can be easily obtained using computerized procedures. Features that are intrinsic to computerized tests that confer benefits over paper-and-pencil measures include millisecond timing accuracy, reliable and randomized presentation of stimuli over multiple trials and repeated administration, and the unobtrusive measurement of cognitive skills and response times during all aspects of the assessment process. Computerized batteries of this type can record aspects of performance that are difficult for psychometrists to measure, and these may reflect the activity of developing neural networks with more sensitivity than can be achieved with traditional tests (Berger, 2006; Luciana, 2003; Schatz \& Browndyke, 2002; Strauss, Sherman, \& Spreen, 2006).

The use of computers in neuropsychological evaluation requires attention and care from the experimenter or clinician. Potential errors in computerized neuropsychological testing could arise because of hardware and software interactions (e.g., resolution of the display and presentation of stimuli affected by the monitor type and size; Cernich, Brennana, Barker, \& Bleiberg, 2007). Computers are helpful in the calculations of scores or conversion from raw to standard scores, but automated scoring has limited usefulness in neuropsychological practice if other variables are not controlled. The importance of paying attention to the patient's response style, manner of dealing with difficulties, type of interaction established, and other important sources of clinically relevant hypotheses has been stressed (Berger, 2006). The analysis of performance by computers needs to be interpreted in the context of psychometric data together with aspects of the patient's history and findings from clinical or neurological examinations (Luciana, 2003).

The normative data for CANTAB have been obtained from over 2000 studies with normal subjects aged 4 to 90 years who participated in several studies conducted primarily in the United Kingdom (Cambridge Cognition, 2006).

The 22 tests that constitute CANTAB (CANTABeclipse) examine various areas of cognitive function including visual memory, visual attention, learning and memory, working memory, planning, set-shifting, sustained attention, and fluid intelligence. The tests are divided into six main groups: motor and visual memory, executive function, working memory and planning, attention, verbal/semantic memory, and decision making and response control (Cambridge Cognition, 2006).

CANTAB allows the investigator to create his/her own battery using a selection of tests tailored to his/her research interest. The battery was first commercialized in 1994. Since then, it has been used by more than 500 academic institutions worldwide and cited in over 600 scientific papers (Cambridge Cognition, 2006).

In this wide range of studies, we can find studies that applied CANTAB in children. Several studies compared controls and a given pathology or specific groups. Examples are the assessment of neuropsychological function after admission to pediatric intensive care (Elison, Shears, Nadel, Sahakian, \& Garralda, 2008), executive function with regard to inhibition, planning, flexible shifting of actions, and working memory in children with high-functioning autism and attention deficit/hyperactivity disorder (Goldberg et al., 2005), neuropsychological performance in children with bipolar disorder (Dickstein et al., 2004), and the effects of a drug (e.g., methylphenidate) on performance in children with attention deficit/hyperactivity disorder (DeVito et al., 2009). Control groups composed of age- and educational level-matched healthy children were employed in these and other studies. However, studies dedicated to obtaining normative values in children are scarce. We found only two such studies (De Luca et al., 2003; Luciana \& Nelson, 2002). De Luca and colleagues described the development of executive function over the lifespan in participants who ranged in age from 8 to 64 years. In children, they found functional gains in the efficiency of working memory capacity, planning, and problemsolving ability. Luciana and Nelson (2002) assessed CANTAB performance in children aged 4 to 12 years. CANTAB has been reported to have high applicability in the evaluation of children with weak verbal skills such as those associated with learning disability, autism, or both.

The neurological disorders investigated in the present report (i.e., multiple sclerosis [MS] and Duchenne muscular dystrophy [DMD]) are known to affect neuropsychological function (Balsimelli, Mendes, Bertolucci, \& Tilbery, 2007; Beatty, Goodkin, Monson, \& Beatty, 1989; Cyrulnik et al., 2008; D’Angelo \& Bresolin, 2006; De Luca, Chelune, Tulsky, Lengenfelder, \& Chiaravalloti, 2004; Desguerre et al., 2009; Dorman, Hurley, \& D'Avignon, 1988; Giliberto, Ferreiro, Dalamon, \& Szijan, 2004; Grant, McDonald, Trimble, Smith, \& Reed, 1984; Hinton, De Vivo, Nereo, Goldstein, \& Stern, 2001; Laatu, Revonsuo, Hämäläinen, Ojanen, \& Ruutiainen, 2001; Miller, Tunnecliffe, \& Douglas, 1985; Moizard et al., 1998; Parmenter, Schucerd, Benedict, \& Schucard, 2006; Parmenter, Shucard, \& Shucard, 2007; Plohmann et al., 1998; ; Rao, 2004; Ruggieri et al., 2003; Santiago, Guardia, Casado, Carmona, \& Arbizu, 2007; Vleugels et al., 2000, 2001).

Multiple sclerosis is an autoimmune inflammatory disease characterized by demyelination and degeneration of the central nervous system. Disease onset usually occurs in young adults and is two- to three-times more frequent in women than men. The average age of onset is between 20 and 40 years, but the disorder may develop at any age.

Duchenne muscular dystrophy is an X-linked form of muscular dystrophy characterized by progressive skeletal 
muscle weakness and irreversible degeneration (Cyrulnik et al., 2008). It is caused by mutations in the dystrophin gene. This gene codes for the dystrophin protein, which is important for maintaining the stability of muscle-fiber membranes (Muntoni, Torelli, \& Ferlini, 2003).

No studies have used CANTAB in DMD patients, and only one group used the battery in MS patients (Foong et al., 1997, 1998, 1999). used subtests of the CANTAB battery (i.e., spatial span, spatial working memory and Tower of London), reporting a significant difference in spatial span between MS patients and controls. In spatial working memory, the patients also had poorer performance compared with the control group. In the Tower of London (Stockings of Cambridge [SOC]) subtest, motor initiation and execution times were significantly longer in patients compared with controls.

Intellectual skills, verbal function, and language of DMD patients have been widely reported in the literature. However, there is a lack of visuo-spatial assessment. This is very relevant because DMD patients have recently been shown to present losses in several visual functions (Costa, Oliveira, Feitosa-Santana, Zatz, \& Ventura, 2007).

Although CANTAB has been used in several countries in different types of research, no studies have used the battery in Brazil. Our objective was to describe the applicability of CANTAB to different samples of Brazilian subjects. Therefore, we present a summary of its application and use in a group of healthy children and two samples of patients - relapsing-remitting MS (RRMS) and DMD — in a detailed descriptive form.

Data were collected at the Laboratório da Visão, Institute of Psychology, University of São Paulo. The procedures were approved by the Human Research Ethics Committee of the University Hospital of the University of São Paulo, and written informed consent was obtained from the participants or their guardians before testing. The statistical analysis was performed using Statistica 9.0 software (StatSoft) for the descriptive statistics, one-way analysis of variance (ANOVA) and analysis of covariance (ANCOVA) for group comparisons, and the Pearson correlation test for correlational analyses. Statistical significance was defined as $p<.05$.

\section{Sample 1: Preliminary norms}

A sample of 40 children aged 6 to 11 years (8.5 \pm 1.7 years) was assessed (17 males and 23 females). The children were recruited from Escola de Aplicação (Education School, University of São Paulo). The exclusion criteria for the children were a history of epilepsy, brain injury, or psychiatric illnesses, evidence of developmental disorder, and an intelligence score below the 25 th percentile measured by Raven's Progressive Matrices Test. Twentythree children had an intelligence score in the 80th percentile or higher (mean, 79th percentile; median, 80th percentile).

\section{Sample 2: Multiple sclerosis patients}

A group of 29 patients ( 7 males and 23 females) were recruited from the Department of Neurology of the Hospital of Clínicas, Faculdade de Medicina da Universidade de São Paulo. They were aged 1854 years $(35.57 \pm 9.85$ years $)$ and had 9 to 15 years of education $(13.62 \pm 3.60)$. All of the patients had been clinically diagnosed with MS according to Poser criteria (Poser \& Brinar, 2001) and classified as having the RRMS.

To participate in this study, the MS patients had bestcorrected visual acuity of 20/20 or better and an EDSS < 2. The Kurtzke Expanded Disability Status Scale (EDSS) is a method of quantifying disability in MS (Kurtzke, 1983). It quantifies disability in eight functional systems. The EDSS scale ranges from 0 to 10 in 0.5 unit increments that represent higher levels of disability. Our patients had minimal disability in only one functional system. The exclusion criteria included the following: less than 8 years of education, a history of head injury, other diseases, and suspected alcohol or drug abuse.

The results were compared with 46 healthy subjects (29 males and 17 females) aged $18-54$ years $(39.5 \pm 12.1$ years $)$ with 9 to 20 years of education $(14.3 \pm 3.5)$. Control subjects were also matched with regard to educational background.

\section{Sample 3: Duchenne muscular dystrophy patients}

Thirty-four subjects with DMD were recruited from the Associação Brasileira de Distrofia Muscular (ABDIM), and 34 controls participated. Both groups were composed of males aged 6 to 26 years. Twenty-nine DMD patients $(85 \%)$ were wheelchairdependent. No significant difference was found between the groups with regard to age $(p=.09)$. The inclusion criterion was an intelligence score within normal limits. Twenty-three DMD subjects and all of the controls had an intelligence quotient (IQ) of $\geq 80$ based on the Wechsler scale. Eleven DMD patients were unable to complete the Wechsler performance tests because of severe motor disability and were assessed with Raven's Progressive Matrices Test. Their minimum 25th percentile was within the normal range. The Raven's percentile was converted to an IQ value (Strauss et al., 2006).

A history of epilepsy, brain injury, or evidence of developmental disorders were exclusion criteria for both groups. Because the DMD patients had significantly lower intelligence scores ( $F=12.7, p<.01$, one-way ANOVA), IQ scores were added to the analysis as a covariate.

The tests were performed using a mouse instead of a touch screen because of motor impairment in the DMD patients. The control group was similarly assessed. The demographic variables of the three samples are presented in Table 1. 


\section{CANTAB subtests}

Nine CANTAB subtests were selected. Tasks that measure working memory (Spatial Span [SSP]) and visual memory (Pattern Recognition Memory [PRM]) were administered to all of the groups. The remaining tests were applied as the following: tasks of sustained attention (Rapid Visual Processing [RVP]) and spatial

Table 1. Demographic variables of each sample

\section{Children's Sample}

$n$

Age (range)

Age (mean)

Age (standard deviation)

Intelligence score (percentile mean)

Intelligence score (percentile standard deviation)

Intelligence score (percentile median)

Proportion (males:females)
40

6-11 years

8.5 years

1.7 years

79

21

90

$17: 23$

\begin{tabular}{|c|c|c|}
\hline RRMS Sample & Patients & Controls \\
\hline$n$ & 29 & 46 \\
\hline Age (range) & $18-54$ years & $18-54$ years \\
\hline Age (mean) & 35.57 years & 39.47 years \\
\hline Age (standard deviation) & 9.83 years & 12.15 years \\
\hline Years of education (range) & $9-15$ years & $9-20$ years \\
\hline Years of education (mean) & 13.62 years & 14.28 years \\
\hline Years of education (standard deviation) & 3.60 years & 3.53 years \\
\hline Proportion (males:females) & $7: 23$ & $27: 17$ \\
\hline DMD Sample & Patients & Controls \\
\hline$n$ & 34 & 34 \\
\hline Age (range) & $6-26$ years & $6-26$ years \\
\hline Age (mean) & 35.57 years & 39.47 years \\
\hline Age (standard deviation) & 9.83 years & 12.15 years \\
\hline Proportion (males:females) & 01:00 & 01:00 \\
\hline IQ (mean) & 95.6 & 113.5 \\
\hline IQ (standard deviation) & 8.6 & 11.1 \\
\hline
\end{tabular}


memory (Spatial Recognition Memory [SRM]) in the neurological groups and visual perception/visual memory (Delayed Matching to Sample [DMS]) in the healthy children and DMD group. RRMS patients and healthy children completed a test of inhibitory control (Stop Signal Task [SST]). Tests of visual planning (SOC) and set shifting (Intra-Extra Dimensional Set Shift [IED]) were administered to the children and a task that measures decision making (Information Sampling Task [IST]) was used in the patients with RRMS.

\section{Results and Discussion}

Sample 1: preliminary norms

We found positive correlations between spatial memory span and age in the working memory task (SSP) for the forward mode $(r=0.61, p=0.00003$; Figure 1) and backward mode $(r=0.49, p=.0026$; Figure 2$)$.

For visual memory, the DMS task showed a positive correlation between hits (percent correct) and age $(r=$ $0.46, p=.0036$ ). In the inhibitory control (SST) task, we found a negative correlation between latency on go trials and age $(r=-0.55, p=.0022)$.

In the visual planning (SOC), set shifting (IED), and visual memory (PRM) tests, we did not find significant correlations with age (Table 2).

\section{Sample 2}

Significant differences were found between control and MS patients in the PRM test that evaluates immediate $(p<.01)$ and delayed $(p<.01)$ visual memory, SSP test that evaluates forward $(p<.01)$ and backward $(p<$

\section{Spatial Span Forward (SSP)}

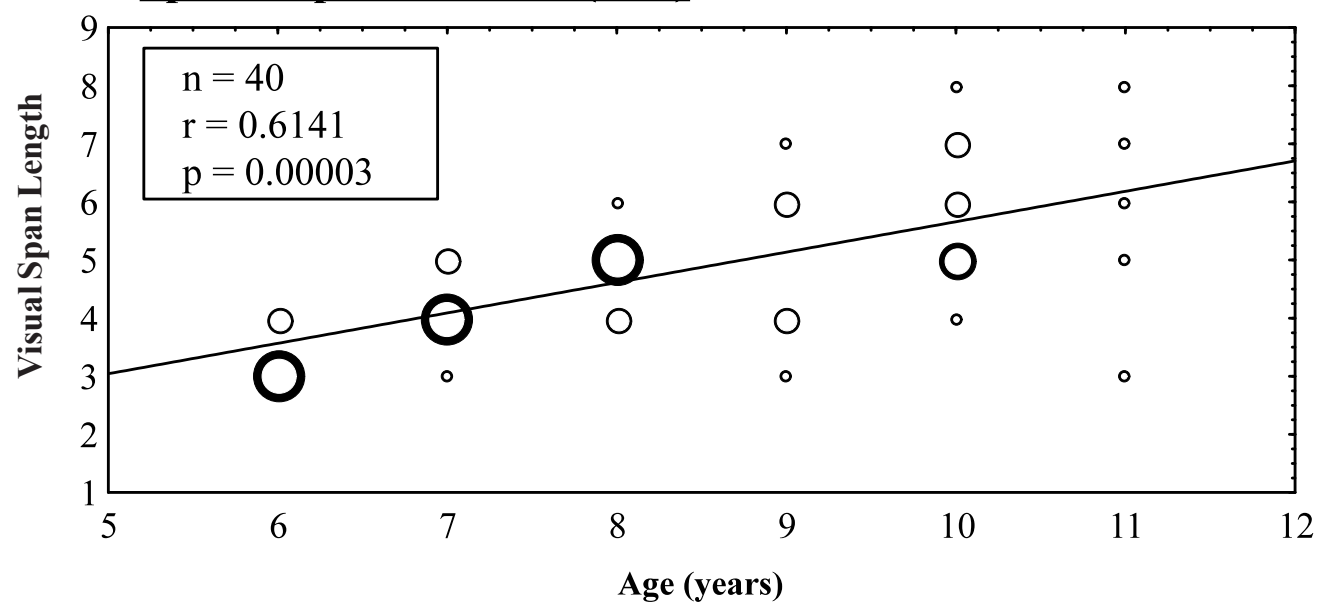

Figure 1. Correlation between working memory and age for forward visual span length. The size of the circles represents the number of children at each data point.

\section{Spatial Span Backward (SSP)}

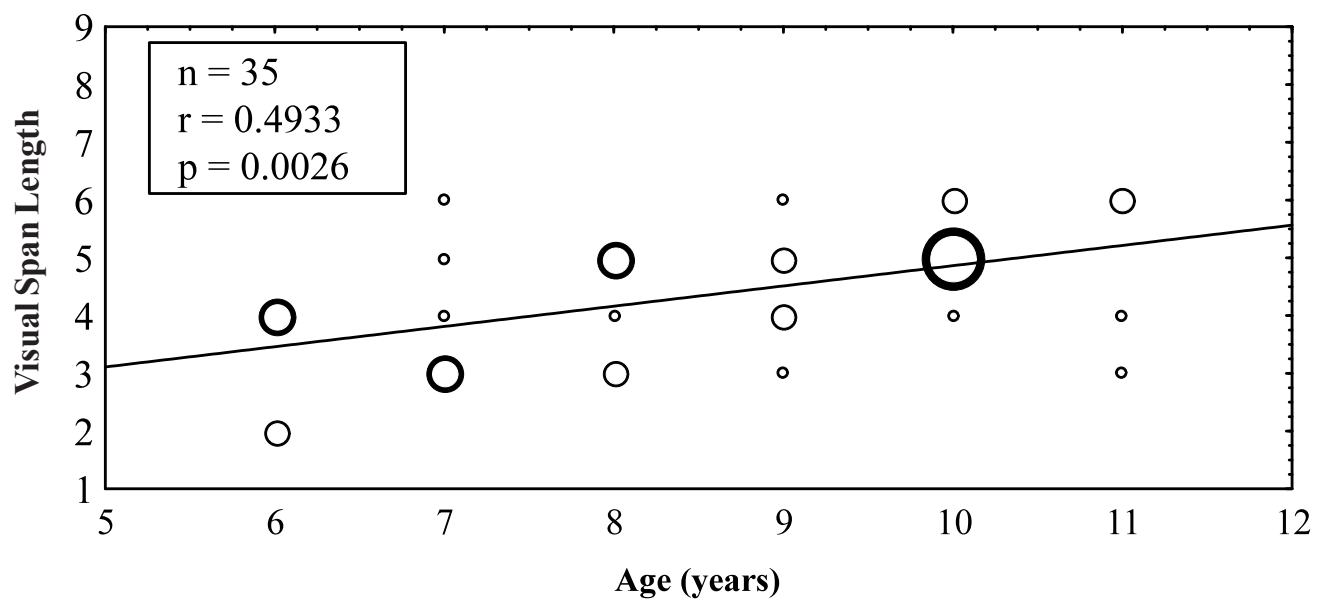

Figure 2. Correlation between working memory and age for backward visual span length. The size of the circles represents the number at each data point. 
Table 2. Correlations between age and test results for children

\begin{tabular}{|c|c|c|c|c|}
\hline Test Results & $n$ & Mean (SD) & $r$ & $p$ \\
\hline \multicolumn{5}{|l|}{ Delayed Matching to Sample } \\
\hline Percent correct (all delays) & 38 & $75.8(13.6)$ & 0.46 & $<0.01$ \\
\hline \multicolumn{5}{|l|}{ Spatial Span } \\
\hline Span length (forward) & 40 & $4.9(1.4)$ & 0.61 & $<0.01$ \\
\hline Span length (backward) & 35 & $4.3(1.1)$ & 0.49 & $<0.01$ \\
\hline \multicolumn{5}{|l|}{ Stop Signal Task } \\
\hline Reaction time on go trials (ms) & 29 & $585(161)$ & -0.55 & $<0.01$ \\
\hline \multicolumn{5}{|l|}{ Stocking of Cambridge } \\
\hline Problems solved in minimum moves & 36 & $5.8(1.8)$ & 0.31 & 0.07 \\
\hline \multicolumn{5}{|l|}{ Intra-Extra Dimensional Set Shift } \\
\hline Stages completed & 33 & $7.7(0.9)$ & 0.10 & 0.58 \\
\hline \multicolumn{5}{|l|}{ Pattern Recognition Memory } \\
\hline Percent correct & 33 & $86.5(10.2)$ & 0.05 & 0.77 \\
\hline
\end{tabular}

$\mathrm{SD}$, standard deviation; ms, milliseconds.

.01) working memory, SRM test that evaluates spatial memory $(p<.01)$, IST test that evaluates decision making latency in the fixed win condition $(p<.01)$ in which the subject is awarded 100 points for a correct decision regardless of the number of boxes opened, and decreasing win condition $(p<.01)$ in which the number of points that can be won for a correct decision starts at 250 and decreases by 10 points for every box touched, SST test that evaluates inhibitory control $(p=.03)$, and RVP test that evaluates sustained attention $(p=.03)$ reflected by reaction time (Table 3 ).

\section{Sample 3}

Duchenne muscular dystrophy patients and controls differed significantly in terms of forward ( $p=$ $.02)$ and backward $(p<.01)$ span on the SSP test and spatial memory $(p<.01)$ on the SRM test (Table 3$)$. The ANCOVA revealed the influence of IQ on these results (as shown by the referred $p$ values). However, $p$ values associated with the disease were not significant $(p>.05)$, suggesting no effect of DMD on the patients' worse performance (Table 4).

\section{Preliminary norms}

The results obtained with the three samples generally agree with both the literature that utilized
CANTAB as a testing tool and the assessments of the same neuropsychological functions performed with other types of tests.

In the children's sample, a comparison between our data and data of Luciana and Nelson (2002) found similar results. The children tested here obtained scores near the mean and within the standard deviation for the visual memory (PRM; Figure 3), working memory (SSP; Figure 4), planning (SOC; Figure 5), and set shifting (IED; Figure 6) tasks.

The results found with the children's sample indicate the high applicability of CANTAB within this group. An increase in SSP performance was found with age, suggesting developmental improvement in visuo-spatial working memory. Our results confirm a previous report that found improvement in SSP performance in children between the ages of 5 years and 11 to 13 years, who reached the results of adults (Nichelli, Bulgheroni, \& Riva, 2001).

For the visual planning (SOC) and set-shifting (IED) subtests, we did not find a correlation between age and performance in the children. Similar results were found by De Luca et al. (2004) and Luciana and Nelson (2002). These findings together suggest the nonlinear development of executive function until maturation at 11 years of age. 
Table 3. Neuropsychological performance in patients and controls of sample 2

\begin{tabular}{|c|c|c|c|c|}
\hline \multirow{2}{*}{ Tests } & \multicolumn{2}{|c|}{ Mean (SD) } & \multirow{2}{*}{$\boldsymbol{F}$} & \multirow{2}{*}{$p$} \\
\hline & MS Group & Control Group & & \\
\hline \multicolumn{5}{|l|}{ Pattern Recognition Memory } \\
\hline Total correct (immediate) & $11(1.81)$ & $11(0.81)$ & 20.2 & $<0.01$ \\
\hline Total correct (delayed) & $9(1.67)$ & $10(1.66)$ & 24.8 & $<0.01$ \\
\hline \multicolumn{5}{|l|}{ Spatial Span } \\
\hline Span length (forward) & $5(0.75)$ & $6(0.75)$ & 16.3 & $<0.01$ \\
\hline Span length (backward) & $5(1.75)$ & $6(1.5)$ & 21.2 & $<0.01$ \\
\hline \multicolumn{5}{|l|}{ Spatial Recognition Memory } \\
\hline Total correct & $12(2.65)$ & $15(2.51)$ & 24.9 & $<0.01$ \\
\hline \multicolumn{5}{|l|}{ Information Sampling Task } \\
\hline Mean Latency -fixed condition & 204 (139) & $155(171)$ & 8.75 & $<0.01$ \\
\hline Mean Latency - decreasing condition & $155(80)$ & $129(88)$ & 8.67 & $<0.01$ \\
\hline \multicolumn{5}{|l|}{ Stop Signal Task } \\
\hline Proportion of successful stops & $0.38(0.2)$ & $0.56(0.1)$ & 4.68 & $<0.04$ \\
\hline \multicolumn{5}{|l|}{ Rapid Visual Processing } \\
\hline Reaction time (ms) & $405(131)$ & $341(86)$ & 5.31 & $<0.04$ \\
\hline
\end{tabular}

In the visual memory subtests, we found interesting results. The PRM subtest did not show a correlation between age and performance, but the DMS subtest showed this correlation. These subtests differ in their measurement of visual memory. The DMS subtest utilizes complex stimuli in which shape, color, and their combination are relevant for discrimination. In the PRM subtest, the relevant aspect of the stimulus is only the

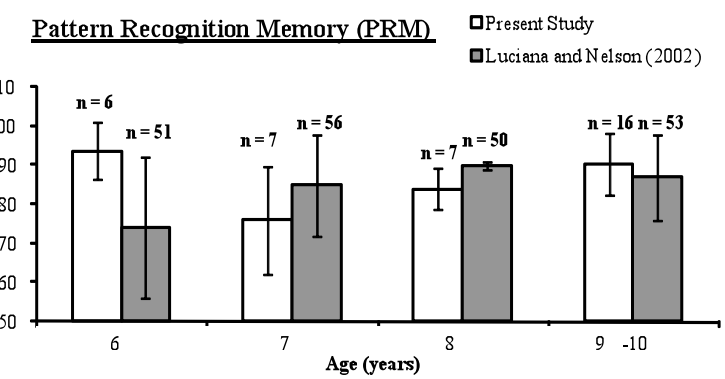

Figure 3. Percentage of correct responses in the CANTAB PRM test in children from 6 to 11 years of age. A comparison is shown between the results from the present study and data from Luciana and Nelson (2002). shape; the color aspect is not relevant for discrimination. The greater complexity of DMS stimuli requires more attention to memorize and recognize the pattern in the future discrimination. Therefore, the DMS correlation with age reflects the development of attention with age, whereas the aspect of visual memory assessed by the PRM subtest does not exhibit a developmental trend in the 6- to 11-year age range.

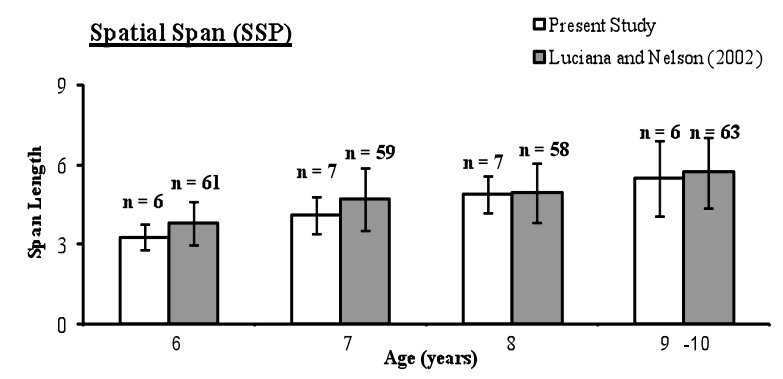

Figure 4. Span length in the CANTAB SSP test in children from 6 to 11 years of age. A comparison is shown between the results from the present study and data from Luciana and Nelson (2002). 
Table 4. Neuropsychological performance in patients and controls of sample 3

\begin{tabular}{|c|c|c|c|c|c|c|}
\hline \multirow{2}{*}{ Tests } & \multicolumn{2}{|c|}{ Mean (SD) } & \multicolumn{2}{|c|}{ Group Effect } & \multicolumn{2}{|c|}{ IQ Effect } \\
\hline & MS Group & Control Group & $\boldsymbol{F}$ & $p^{* *}$ & $\boldsymbol{F}$ & $p^{* *}$ \\
\hline \multicolumn{7}{|c|}{ Pattern Recognition Memory } \\
\hline Total correct (immediate) & $11(1.42)$ & $11(1.22)$ & 0.88 & 0.35 & 1.84 & 0.17 \\
\hline Total correct (delayed) & $9(3.0)$ & $10(1.75)$ & 0.06 & 0.81 & 3.48 & 0.08 \\
\hline \multicolumn{7}{|l|}{ Spatial Span } \\
\hline Span length (forward) & $5(1.22)$ & $7(1.50)$ & 2.74 & 0.10 & 5.84 & 0.02 \\
\hline Span length (backward) & $4(1.11)$ & $6(2.0)$ & 0.01 & 0.90 & 9.36 & $<0.01$ \\
\hline \multicolumn{7}{|c|}{ Spatial Recognition Memory } \\
\hline Total correct & $12(3.34)$ & $14(3.0)$ & 0.12 & 0.72 & 15.8 & $<0.01$ \\
\hline \multicolumn{7}{|c|}{ Delayed Matching to Sample } \\
\hline Total correct (all delays) & $16(3.0)$ & $17(2.25)$ & 0.34 & 0.56 & 2.25 & 0.14 \\
\hline \multicolumn{7}{|l|}{ Rapid Visual Processing } \\
\hline Reaction time (ms) & $353(100)$ & $341(107)$ & 0.13 & 0.71 & 1.34 & 0.25 \\
\hline
\end{tabular}

*ANOVA, **ANCOVA.

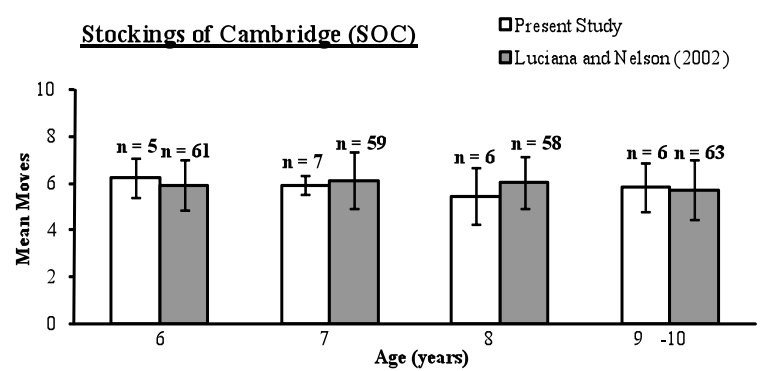

Figure 5. Mean moves (4-move problems) in the CANTAB SOC test in children from 6 to 11 years of age. A comparison is shown between the results from the present study and data from Luciana and Nelson (2002).

In the inhibitory control task (SST), the negative correlation between reaction time and age indicated an improvement in the responses, reflecting development in motor and processing skills in the ages assessed.

Luciana and Nelson (2002) discussed children's vulnerability to fatigue and boredom and included a break between tasks according the child's need. To avoid these problems, our evaluation of children was divided into two or three sessions, and a break between subtests was used when necessary. The comparison between our data and data presented by Luciana and Nelson (2002) in several

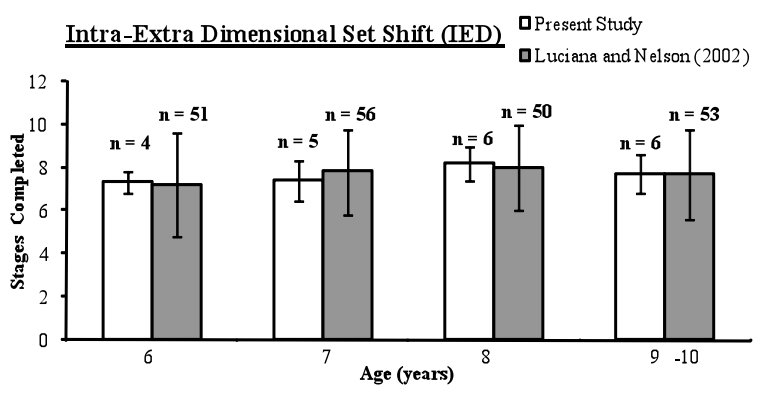

Figure 6. Stages completed in the CANTAB IED test in children from 6 to 11 years of age. A comparison is shown between the results from the present study and data from Luciana and Nelson (2002).

CANTAB tasks showed similar results, despite the language and cultural differences between our samples.

However, independent of the ease of application of CANTAB, establishing good rapport with children is important in the evaluation. The examiner should take a few minutes to talk to the child about play, school, or any subject that is interesting to the child. If good rapport is established with the child, then they more easily engage in the test.

\section{Multiple sclerosis}

In the MS sample, the present results agree with 
those of previous studies that also showed impairment in visual memory (Beatty et al., 1989; Grant et al., 1984; Ruggieri et al., 2003), working memory (Parmenter et al., 2006; Santiago et al., 2007), visuo-spatial function (Laatu et al., 2001; Rao, 2004; Vleugels et al., 2000, 2001), and information processing speed (Balsimelli et al., 2007; De Luca et al., 2004; Parmenter et al., 2007; Plohmann et al., 1998).

Cognitive deficits may affect $40-65 \%$ of MS patients and significantly contribute to their disability (Amato, Zipoli, \& Portaccio, 2006). The cognitive domains most commonly impaired are memory and learning, attention, executive function, and visuo-spatial ability (Rao, Leo, Bernardin, \& Unverzagt, 1991).

In the early stages of MS, the patient already suffers deficits in the main areas of memory, including shortterm memory, learning, and long-term memory, with the verbal and nonverbal domains equally affected (Grant et al., 1984). We confirmed these findings in the present study in which MS patients showed impairment in both the immediate and delayed recall tests.

In the SSP, a computerized version of the Corsi Block Tapping Task, which assesses the ability to remember a sequence of squares lit up on the screen (forward and backward), a significant difference was found between patients and controls, a result similar to that of Foong et al. (1997).

The patients also showed impairment in inhibitory control, reaction time, and response latency in decision making. These findings are all attributable to the fact that the speed of information processing in MS patients is greatly impaired (De Luca et al., 2004; Parmenter, et al., 2007).

Our results showed that cognitive decline was present even when physical disability was not yet severe, but it was mild and did not limit the patients' ability to work as previously reported by Ruggieri et al. (2003). Glanz, Healy, Hviid, Chitnis, and Weiner (2011) observed a deterioration in working memory and visual spatial memory in subjects with early MS over a period of up to 5 years.

Our results are similar to those of Ruggieri et al. (2003) who found that cognitive decline was present in MS patients even before the decline in physical function. A more recent study by Glanz et al. (2011) showed that individuals who had the disease for less than 5 years already presented a decline in working memory, visual memory, and spatial memory.

\section{Duchenne muscular dystrophy}

CANTAB scores in DMD patients indicated impairment in working memory and spatial memory. To our knowledge, this is the first time that patients with DMD were assessed with CANTAB.

Similar to the previous two samples, the present results in DMD patients agree with studies that reported deficits in verbal working memory (Bresolini et al., 1994; Hinton, De Vivo, Fee, Goldstein, \& Stern, 2000;
Hinton et al., 2001; Hinton, De Vivo, Fee, Goldstein, \& Stern, 2004; Hinton, Fee, Goldstein, \& De Vivo, 2007; Wicksell, Kihlgren, Melin, \& Eeg-Olofsson, 2004). One investigation included young children aged 3 to 6 years and found visuospatial working memory impairment (Cyrulnick et al., 2008).

The DMD population presents lower IQs and higher rates of mental retardation (D'Angelo \& Bresolin, 2006; Felisari et al., 2000; Giliberto et al., 2004). With regard to memory, several studies that used non-computerized tests support the impairment of verbal working memory in DMD patients (Bresolini et al., 1994; Hinton et al., 2000, 2001, 2004, 2007; Wicksell et al., 2004). Our findings showed a loss of memory in the visual domain, with impaired performance in the SSP subtest. We suggest that a general working memory deficit may be present in these patients. Although the patients had normal intelligence scores, only IQ emerged as a predictor of deficits in spatial memory and working memory. This finding suggests a primary intelligence deficit that underlies other neuropsychological deficits in patients with DMD.

Despite the influence of IQ on neuropsychological performance, rather than the influence of the disease, the strength of this descriptive approach is that CANTAB was useful with this patient sample and sensitive to specific deficits. The use of the mouse instead of the touch screen in the adaptation to the patient's motor limitations was appropriate and enables their assessment because DMD patients are unable to complete various regular visuospatial neuropsychological tests.

\section{General comments about CANTAB}

The CANTAB battery is particularly appropriate for assessing various diseases for which early detection is very important. Moreover, the wide range of data already collected in several countries gives this battery a very reliable database. Additionally, the applicability of experimental tests developed for animal research (Strauss et al., 2006) to humans opens the way for studies in children with special difficulties because of the easy application of the test.

As mentioned by Luciana and Nelson (2002), the fact that CANTAB tests are mostly non-verbal enables its administration in many countries and makes it independent of language and culture. Its testability in different samples in the present study allows us to confirm the applicability of CANTAB in these samples of the Brazilian population.

Computers should be incorporated in the practice of neuropsychology, but care should be taken with their use. It is essential to pay attention to problems that can occur with both hardware and software. Furthermore, computer-calculated scores are simply numbers. Interpretations need to be made with a contextual comprehension of the patient (e.g., history, 
clinical findings, etc.), and this type of interpretation and understanding cannot be made by a computer.

CANTAB exhibited many advantages in our studies. Its easy administration enables its application in different samples, controls, and patients with neurodegenerative conditions, with little change in the standard procedure, such as using a mouse instead of a touch screen for testing subjects with motor limitations. It also can be used with different age groups (i.e., in both children and adults), allowing the evaluation of children as young as 6 years old.

CANTAB's design allows its use in subjects with weak interaction skills, such as very young children, for whom the user-friendly format is especially helpful because it entertains them and maintains their attention during the test. The battery also offers a pretest phase for some subtests. Similar to a training phase, this stage provides the experimenter an opportunity to verify the subject's understanding of the instructions and to clarify the procedure with more detailed information if necessary. For children, the SSP backward mode was applied after the SSP forward mode. The pretest phase was quite important for these subjects because when they see the same arrangement on the screen again, they quickly respond similarly to the first time (forward mode), despite the new instructions.

Studies that utilize CANTAB may lead to the development of specific protocols that are sensitive to conditions that can impair neural function. Further work should include larger samples and develop reliability studies.

\section{Acknowledgment}

This work was supported by grants from the Foundation for Research Support of the State of São Paulo (FAPESP) Projeto Temático 02/12733-8 (DFV), Doctoral Fellowship (05/53823-8; ECZ), and Undergraduate Fellowship (08/590001-8; DTR). RAAT received a Doctoral Fellowship from the National Council for Scientific and Technological Development (CNPq). DFV has a CNPq 1A Research Fellowship.

\section{References}

Amato, M.P., Zipoli, V., \& Portaccio, E. (2006). Multiple sclerosis-related cognitive changes: a review of cross-sectional and longitudinal studies. Journal of Neurological Science, 245(1-2), 41-46.

Balsimelli, S., Mendes, M.F., Bertolucci, P.H.F., \& Tilbery, C.P. (2007). Attention impairment associated with relapsing-remitting multiple sclerosis patients with mild incapacity. Arquivos de Neuropsiquiatria, 65, 262-267.

Beatty, W.W., Goodkin, D.E., Monson, N., \& Beatty, P.A. (1989). Cognitive disturbances in patients with relapsing remitting multiple sclerosis. Archives of Neurology, 46, 1113-1119.

Berger, M. (2006). Computer assisted clinical assessment. Child and Adolescent Mental Health, 11, 64-75.

Bresolin, N., Castelli, E., Comi, G.P., Felisari, G., Bardoni, A., Perani, D., ... Scarlato, G. (1994). Cognitive impairment in Duchenne muscular dystrophy. Neuromuscular Disorders, 4, 359-369.

Cambridge Cognition (2006). Neuropsychological Test Automated
Battery (CANTABeclipse) manual. Cambridge: Cambridge Cognition Limited.

Cernich, A.N., Brennana, D.M., Barker, L.M., \& Bleiberg, J. (2007). Sources of error in computerized neuropsychological assessment. Archives of Clinical Neuropsychology, 22(Suppl. 1), S39-S48.

Costa, M.F., Oliveira, A.G.F., Feitosa-Santana, C., Zatz, M., \& Ventura, D.F. (2007). Red-green color vision impairment in Duchenne muscular dystrophy. American Journal of Human Genetics, 80, 1064-1075.

Cyrulnik, S.E., Fee, R.J., Batchelder, A., Kiefel, J., Goldstein, E., \& Hinton, V.J. (2008). Cognitive and adaptive deficits in young children with Duchenne muscular dystrophy (DMD). Journal of the International Neuropsychological Society, 14, 853-861.

D'Angelo, M.G., \& Bresolin, N. (2006). Cognitive impairment in neuromuscular disorders. Muscle and Nerve, 34, 16-33.

De Luca, J., Chelune, G.J., Tulsky, D.S., Lengenfelder, J., \& Chiaravalloti, N.D. (2004). Is speed of processing or working memory the primary information processing deficit in multiple sclerosis? Journal of Clinical and Experimental Neuropsychology, $26,550-562$

De Luca, C.R., Wood, S.J., Anderson, V., Buchanan, J.A., Proffitt, T.M., Mahony, K., \& Pantelis, C. (2003). Normative data from the CANTAB: I. Development of executive function over the lifespan. Journal of Clinical and Experimental Neuropsychology, 25, 242-254.

Desguerre, I., Christov, C., Mayer, M., Zeller, R., Becane, H.M., Bastuji-Garin, S., ...Gherandi, R.K. (2009). Clinical heterogeneity of Duchenne muscular dystrophy (DMD): definition of sub-phenotypes and predictive criteria by long-term follow-up. PLoS One, 4, e4347.

DeVito, E.E., Blackwell, A.D., Clark, L., Kent, L., Dezsery, A.M., Turner, D.C., Aitken, M.R.F., \& Sahakian, B.J. (2009) Methylphenidate improves response inhibition but not reflectionimpulsivity in children with attention deficit hyperactivity disorder (ADHD). Psychopharmacology, 202, 531-539.

Dickstein, D.P., Treland, J.E., Snow, J., McClure, E.B., Mehta, M.S., Towbin, K.E., Pine, D.S., \& Leibenluft, E. (2004) Neuropsychological performance in pediatric bipolar disorder. Biology Psychiatry, 55(1), 32-39.

Dorman, C., Hurley, A.D., \& D'Avignon, J. (1988). Language and learning disorders of older boys with Duchenne muscular dystrophy. Developmental Medicine and Child Neurology, 30, 316-327.

Elison, S., Shears, D., Nadel, S., Sahakian, B., \& Garralda, M.E. (2008). Neuropsychological function in children following admission to pediatric intensive care: a pilot investigation. Intensive Care Medicine, 34, 1289-1293.

Felisari, G., Martinelli Boneschi, F., Bardoni, A., Sironi, M., Comi, G.P., Robotti, M., ... Bresolin, N. (2000). Loss of Dp140 dystrophin isoform and intellectual impairment in Duchenne dystrophy. Neurology, 55, 559-564.

Foong, J., Rozewicz, L., Davie, C.A., Thompson, A.J., Miller, D.H., \& Ron, M.A. (1999). Correlates of executive function in multiple sclerosis: the use of magnetic resonance spectroscopy as an index of focal pathology. Journal of Neuropsychiatry and Clinical Neurosciences, 11(1), 45-50.

Foong, J., Rozewicz, L., Quaghebeur, G., Davie, C.A., Kartsounis, L.D., Thompson, A.J., Miller, D.H., \& Ron, M.A. (1997). Executive function in multiple sclerosis: the role of frontal lobe pathology. Brain, 120, 15-26.

Foong, J., Rosewicz, L., Quaghebeur, G., Thompson, A.J., Miller, D.H., \& Ron, M.A. (1998). Neuropsychological deficits in multiple sclerosis after acute relapse. Journal of Neurology, Neurosurgery \& Psychiatry, 64, 529-532.

Fray, P.J., Robbins, T.W., \& Sahakian, B.J. (1996). Neuropsychiatric applications of CANTAB. International Journal of Geriatric Psychiatry, 11, 329-336.

Glanz, B.I., Healy, B.C., Hviid, L.E., Chitnis, T., \& Weiner, H.L. (in press). Cognitive deterioration in patients with early multiple sclerosis: a 5-year study. Journal of Neurology, Neurosurgery and Psychiatry.

Giliberto, F., Ferreiro, V., Dalamon, V., \& Szijan, I. (2004). Dystrophin deletions and cognitive impairment in Duchenne/Becker muscular dystrophy. Neurological Research, 26(1), 83-87.

Goldberg, M.C., Mostofsky, S.H., Cutting, L.E., Mahone, E.M., Astor, B.C., Denckla, M.B., \& Landa, R.J. (2005). Subtle executive impairment in children with autism and children with ADHD. Journal of Autism and Developmental Disorders, 35, 279-293.

Grant, I., McDonald, W.I., Trimble, M.R., Smith, E., \& Reed, R. (1984). Deficient learning and memory in early and middle phases 
of multiple sclerosis. Journal of Neurology, Neurosurgery and Psychiatry, 47, 250-255.

Hadwin, J.A., Brogan, J., \& Stevenson, J. (2005). State anxiety and working memory in children: a test of processing efficiency theory. Educational Psychology, 25, 379-393.

Hinton, V.J., De Vivo, D.C., Fee, R., Goldstein, E., \& Stern, Y. (2000). Investigation of poor academic achievement in children with Duchenne muscular dystrophy. Learning Disabilities Research \& Practice, 19, 146-154.

Hinton, V.J., De Vivo, D.C., Fee, R., Goldstein, E., \& Stern, Y. (2004). Investigation of poor academic achievement in children with Duchenne muscular dystrophy. Learning Disabilities Research \& Practice, 19, 146-154.

Hinton, V.J., De Vivo, D.C., Nereo, N.E., Goldstein, E., \& Stern, Y. (2001). Selective deficits in verbal working memory associated with a known genetic etiology: the neuropsychological profile of Duchenne muscular dystrophy. Journal of the International Neuropsychology Society, 7(1), 45-54.

Hinton, V.J., Fee, R., Goldstein, E.M., \& De Vivo, D.C. (2007). Verbal and memory skills in Duchenne muscular dystrophy. Developmental Medicine and Child Neurology, 49, 123-128.

Kurtzke, J.F. (1983). Rating neurologic impairment in multiple sclerosis: an expanded disability status scale (EDSS). Neurology, $33,1444-1452$.

Laatu, S., Revonsuo, A., Hämäläinen, P., Ojanen, V., \& Ruutiainen, J. (2001). Visual object recognition in multiple sclerosis. Journal of Neurological Science, 185, 77-88.

Levaux, M.N., Potvin, S., Sepehry, A.A., Sablier, J., Mendrek, A., \& Stip, E. (2007). Computerized assessment of cognition in schizophrenia: promises and pitfalls of CANTAB. European Psychiatry, 22, 104-115.

Liotti, M., Pliszka, S.R., Perez, R., 3rd, Luus, B., Glahn, D., \& SemrudClikeman, M. (2007). Electrophysiological correlates of response inhibition in children and adolescents with ADHD: influence of gender, age, and previous treatment history. Psychophysiology, 44, 936-948.

Lowe, C., \& Rabbitt, P. (1998). Test/re-test reliability of the CANTAB and ISPOCD neuropsychological batteries: theoretical and practical issues. Neuropsychologia, 36, 915-923.

Luciana, M. (2003). Computerized assessment of neuropsychological function in children: clinical and research applications of the Cambridge Neuropsychological Testing Automated Battery (CANTAB). Journal of Child Psychology and Psychiatry, 44, 649-663.

Luciana, M., \& Nelson, C.A. (2002). Assessment of neuropsychological function through use of the Cambridge Neuropsychological Testing Automated Battery: performance in 4- to 12-year-old children. Developmental Neuropsychology, 22, 595-624.

Miller, G., Tunnecliffe, M., \& Douglas, P.S. (1985). IQ, prognosis and Duchenne muscular dystrophy. Brain and Development, 7(1), 7-9.

Moizard, M.P., Billard, C., Toutain, A., Berret, F., Marmin N., \& Moraine, C. (1998). Are Dp71 and Dp140 brain dystrophin isoforms related to cognitive impairment in Duchenne muscular dystrophy? American Journal of Medical Genetics, 80(1), 32-41.

Muntoni, F., Torelli, S., \& Ferlini, A. (2003). Dystrophin and mutations: one gene, several proteins, multiple phenotypes. Lancet Neurology, 2, 731-740.

Nichelli, F., Bulgheroni, S., \& Riva, D. (2001). Developmental patterns of verbal and visuospatial spans. Neurological Sciences, $22,377-384$.

Owen, A.M., Sahakian, B.J., Semple, J., Polkey, C.E., \& Robbins, T.W. (1994). Visuo-spatial short-term recognition memory and learning after temporal lobe excisions, frontal lobe excisions or amygdalo-hippocampectomy in man. Neuropsychologia, 33, 1-24.

Palade, C., \& Benga, S. (2007). Neuropsychological impairments on the CANTAB test battery: case reports of children with frontal and temporal lobe epilepsy. Cognition, Brain, Behavior, 11, 539-552.

Pantelis, C., Barnes, T.R.E., Nelson, H.E., Tanner, S., Weatherley, L., Owen, A.M., \& Robbins, T.W. (1997). Frontal-striatal cognitive deficits in patients with chronic schizophrenia. Brain, 120, 1823-1843.

Parmenter B.A., Shucerd J.L., Benedict R.B., \& Schucard D.W. (2006)
Working memory deficits in multiple sclerosis: Comparison between the n-back task and the Paced Auditory Serial Addition Test. Journal of the International Neuropsychological Society, 12, 677-687.

Parmenter, B.A., Shucard, J.L., \& Shucard, D.W. (2007). Information processing deficits in multiple sclerosis: a matter of complexity. Journal of the International Neuropsychological Society, 13, 417-423.

Plohmann, A.M., Kappos, L., Ammann, W., Thordai, A., Wittwer, A., Huber, S., Bellaiche, Y., \& Lechner-Scott, J. (1998). Computer assisted retraining of attentional impairments in patients with multiple sclerosis. Journal of Neurology, Neurosurgery and Psychiatry, 64, 455-462.

Poser, C.M., \& Brinar, V.V. (2001). Diagnostic criteria for multiple sclerosis. Clinical Neurology and Neurosurgery, 103, 1-11.

Rao, S.M. (2004). Cognitive function in patients with multiple sclerosis: impairment and treatment. International Journal of MS Care, 1, 9-22.

Rao, S.M., Leo, G.J., Bernardin, L., \& Unverzagt, F. (1991). Cognitive dysfunction in multiple sclerosis: I. Frequency, pattern, and prediction. Neurology, 41, 685-691.

Rasmussen, C., Soleimani, S., Carroll, A., \& Hodlevskyy, O. (2009). Neuropsychological functioning in children with Tourette syndrome (TS). Journal of the Canadian Academy of Child and Adolescent Psychiatry, 18, 307-315.

Robbins, T.W., James, M., Owen, A.M., Sahakian, B.J., Lawrence, A.D., McInnes, L., \& Rabbitt, P.M.A. (1998). A study of performance on tests from the CANTAB battery sensitive to frontal lobe dysfunction in a large sample of normal volunteers: implications for theories of executive functioning and cognitive aging. Journal of the International Neuropsychological Society, 4, 474-490.

Ruggieri, R.M., Palermo, R., Vitello, G., Gennuso, M., Settipani, N., $\&$ Piccoli, F. (2003). Cognitive impairment in patients suffering from relapsing-remitting multiple sclerosis with EDSS $\leq 3.5$. Acto Neurologica Scandinavica, 108, 323-326.

Sahakian, B.J., Morris, R.G., Evenden, J.L., Heald, A., Levy, R., Philpot, M., \& Robbins, T.W. (1988). A comparative study of visuospatial memory and learning in Alzheimer-type dementia and Parkinson's disease. Brain, 111, 695-718.

Santiago, O., Guardia, J., Casado, V., Carmona, O., \& Arbizu, T. (2007). Specificity of frontal dysfunctions in relapsing-remitting multiple sclerosis. Archives of Clinical Neuropsychology, 22, 623-629.

Schatz, P., \& Browndyke, J. (2002). Applications of computerbased neuropsychological assessment. Journal of Head Trauma Rehabilitation, 17, 395-410.

Steele, S.D., Minshew, N.J., Luna, B., \& Sweeney, J.A. (2007). Spatial working memory deficits in autism. Journal of Autism and Developmental Disorders, 37(4): 605-612.

Strauss, E., Sherman, E.M.S., \& Spreen, O. (2006). A compendium of neuropsychological tests: administration, norms, and commentary, (3rd edition). New York: Oxford University Press.

Sweeney, J.A., Kmiec, J.A., \& Kupfer, D.J. (2000). Neuropsychologic impairments in bipolar and unipolar mood disorders on the CANTAB neurocognitive battery. Biological Psychiatry, 48, 674-684.

Torgersen, J., Helland, C., Flaatten, H., \& Wester, K. (2010). Reversible dyscognition in patients with a unilateral, middle fossa arachnoid cyst revealed by using a laptop based neuropsychological test battery (CANTAB). Journal of Neurology, 257, 1909-1916.

Vleugels, L., Lafosse, C., van Nunen, A., Nachtergaele, S., Ketelaer, P., Chaelier, M., \& Vandenbussche, E. (2000). Visuoperceptual impairment in multiple sclerosis patients diagnosed with neuropsychological tasks. Multiple Sclerosis, 6, 241-254.

Vleugels, L., Lafosse, C., van Nunen, A., Chaelier, M., Ketelaer, P., \& Vandenbussche, E. (2001). Visuoperceptual impairment in MS patients: nature and possible neural origins. Multiple Sclerosis, 7, 389-401.

Wicksell, R.K., Kihlgren, M., Melin, L., \& Eeg-Olofsson, O. (2004). Specific cognitive deficits are common in children with Duchenne muscular dystrophy. Developmental Medicine and Child Neurology, 46, 154-159.

Yun da, Y., Hwang, S.S., Kim, Y., Lee, Y.H., Kim, Y.S., \& Jung, H.Y.(2011). Impairments in executive functioning in patients with remitted and non-remitted schizophrenia. Progress in Neuropsychopharmacology and Biological Psychiatry, 35, 1148-1154. 